\title{
ON THE HADAMARD PRODUCTS OF SCHLICHT FUNCTIONS AND APPLICATIONS
}

\author{
H.S. AL-AMIRI \\ Department of Mathematics \\ Bowling Green State University \\ Bowling Green, Ohio 43403
}

(Received April 25, 1984)

ABSTRACT. We show that each of the schlicht classes of starlike, convex, close-toconvex and strongly starlike with respect to symmetric points is invariant under the Hadamard product with the class of convex functions. The influence of certain operators over these classes is also investigated.

KEY WORDS AND PHRASES. Hadamard products, schlicht functions, and starlike, coriver, close-to convex and strongly starlike with respect to symmetric points. 1980 MATHEMATICS SUBJECT CLASSIFICATION CODE. Primary $30 C 45$

1. INTRODUCTION.

Let $A$ be the class of analytic functions on the unit disk $U=\{z:|z|<1\}$. By $S$ we denote those functions in $A$ which are univalent and normalized by $f(0)=$ $f^{\prime}(0)-1=0$. Let $C, S^{*}$ and $K$ be the usual subclasses of $S$ which are consisting of the convex, starlike and close-to-convex functions, respectively.

The Hadamard product (convolution) of two power series $f(z)=\sum_{0}^{\infty} a_{n} z^{n}$, $g(z)=\sum_{0}^{\infty} b_{n} z^{n}$ is defined as the power series $(f * g)(z)=\sum_{0}^{\infty} a_{n} b_{n} z^{n}$. Pólya and Schoenberg [9] have conjectured that $f * g$ is in $C$ whenever both $f$ and $g$ are in C. This conjecture was finally shown to be true by Ruscheweyh and Sheil-Smal1 [12.). THEOREM A (Ruscheweyh and Shei1-Sma11): Let $h$ be in $C$. If $f$ is in $C, S^{*}$ or $K$ then $\mathrm{h} * \mathrm{f}$ is in $\mathrm{C}, \mathrm{S}^{*}$ or $\mathrm{K}$ respectively.

The proof of Theorem A requires a key lemma that turned out to be beautiful in its simplicity and powerful in its applicability to various problems. We state this key lemma of Ruscheweyh and Sheil-Small in the form stated and proved by Barnard and Kellogg in $[1]$.

LEMMA A. Let $\phi$ and $g$ be analytic in $|z|<1$ with $\phi(0)=g(0)=0$ and $\phi^{\prime}(0) g^{\prime}(0) \neq 0$. Suppose that for each $\alpha(|\alpha|=1)$ and $\sigma(|\sigma|=1)$ we have

$$
\left[\phi *\left(\frac{1+\alpha \sigma z}{1-\sigma z}\right) g\right](z) \neq 0
$$

on $0<|z|-r \cdot 1$. Then for each $F$ in $A$ the image of $|z|<r$ uncler 
$(\phi * \mathrm{Fg}) /(\phi * \mathrm{~g})$ is a subset of the convex hu11 of $\mathrm{F}(\mathrm{U})$.

REMARK 1. In [12], it was shown that condition [1] is satisfied for all $z$ in $U$ whenever $\phi$ is in $C$ and $g$ is in $S^{*}$.

A function $f$. A is said to be starlike with respect to symmetric points in $U$, denoted $\mathrm{f} \in \mathrm{S}_{\mathrm{S}}{ }^{*}$, if and on $1 \mathrm{y}$ if

$$
\operatorname{Re} \frac{z f^{\prime}(z)}{f(z)-f(-z)}>0 \text {, for all } z \text { in } U \text {. }
$$

This class was introduced by Sakaguchi [13].

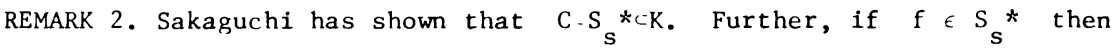
$(f(z)-f(-z)) / 2$ is in $S^{*}$.

We say that $f$ is strongly starlike of order $\lambda$, with respect to symmetric points in $U$, denoted $f \cdot S_{S} *(\lambda)$, if and on $1 y$ if

$$
\left|\arg \left\{\frac{z f^{\prime}(z)}{f(z)-f(-z)}\right\}\right|-\frac{\lambda \pi}{2}, 0<\lambda<1, \quad z \in U .
$$

This class was introduced by Padmanabhan and Thangamant [8].

Das and Singh [4] introduced the classes of function $f$ which are convex with respect to symmetric points in $U$, denoted $f C_{S}$, and those which are close-toconvex with respect to symmetric points, denoted $f \in K_{s}$ as follows: $f C_{s}$ if and only if

$$
\operatorname{Re} \frac{z\left(z f^{\prime}(z)\right)^{\prime}}{z f^{\prime}(z)+z f^{\prime}(-z)}>0, z ; U
$$

and $f r K_{S}$ if and only if there exists $K \in \mathrm{S}_{S}^{*}$ such that

$$
\operatorname{Re} \frac{z f^{\prime}(z)}{K(z)-K(-z)}>0, \quad z ; U \text {. }
$$

REMARK 3. In [4], it was shown that both $C_{s}$ and $K_{s}$ are subsets of $s$ and furthermore, $f \in \mathrm{C}_{S}$ if and only if $\mathrm{zf}^{\prime} \in \mathrm{S}_{\mathrm{S}}{ }^{*}$.

The purpose of this note is to investigate these subclasses of $S$ which are symmetric with respect to points in $U$ via the Hadamard products. In section 2 , we state our main theorem which shows that these classes are invariant under the Hadamard product with convex functions. In section 3, we use the results of section 2 and an observation made in [1] to obtain results concerning the radii of these classes under the influence of certain well known operators.

2. MAIN RESULTS

The elegant technique and results of Ruscheweyh and Sheil-Small allow us to produce a theorem similar to Theorem A for the classes $S_{s}{ }^{*}, C_{s}, K_{s}$ and $s_{s}{ }^{*}(\lambda)$, $0<\lambda<1$.

THEOREM 1. Let $h \in C$. If $f$ is in $S_{s}{ }^{*}, C_{s}, K_{s}$ or $S_{s} *(\lambda), 0<\lambda \leq 1$, then $h * f$ is in $\mathrm{S}_{\mathrm{s}}{ }^{*}, \mathrm{C}_{\mathrm{s}}, \mathrm{K}_{\mathrm{s}}$ or $\mathrm{S}_{\mathrm{s}} *(\lambda)$ respectively.

PROOF. The proof of the four cases requires an application of Lemma A. In order to apply this lemma, we need to identify the convex function $\phi$ and the starlike function $g$ for the different cases. Case 1. Let $\mathrm{f} \in \mathrm{S}_{\mathrm{S}}^{*}$ and $\mathrm{h} \in \mathrm{C}$. Then $\mathrm{g}(\mathrm{z})=\frac{\mathrm{f}(\mathrm{z})-\mathrm{f}(-\mathrm{z})}{2} ; \mathrm{S}^{*}$ by Remark 2 . Thus 
condition (1.1)is satisfied according to Remark 1, i.e.,

$$
\left[h *\left(\frac{1+\alpha \sigma z}{1-\sigma z}\right) g\right](z) \neq 0
$$

for all $z$ in $U$. Now let

$$
F(z)=\frac{z f^{\prime}(z)}{f(z)-f(-z)}
$$

Applying Lemma Aith $F$ as given in(2.1) we obtain

$$
\begin{aligned}
\left(\frac{h * F g}{h * g}\right)(z) & =\frac{h(z) * \frac{z f^{\prime}(z)}{(f(z)-f(-z))} \cdot \frac{(f(z)-f(-z))}{2}}{h(z) *\left(\frac{f(z)-f(-z)}{2}\right)} \\
& =\frac{h(z) * z f^{\prime}(z)}{(h * f)(z)-(h * f)(-z)}=\frac{z(h * f)^{\prime}(z)}{(h * f)(z)-(h * f)(-z)}
\end{aligned}
$$

which is contained in the convex hull of $F$ in $U$. This implies

$$
\operatorname{Re} \frac{z\left(l_{i} * f\right)^{\prime}(z)}{(h * f)(z)-(h * f)(-z)}>0, z \in U,
$$

or $\mathrm{h} * \mathrm{f} * \mathrm{~S}_{\mathrm{S}} *$ according to $(1.2)$.

CASE 2. Let $f \in C_{s}$ and $h \in C$. This case follows immediately from Remark 3 and Case 1 above.

CASE 3. Let $f \in K_{S}$ and $h \in C$. Let $K(z)$ be the associated function appearing in (1.5). Then according to Remark $2 g(z)=\frac{K(z)-K(-z)}{2} \varepsilon S^{*}$. Thus condition (1.1) is satisfied for $h$ and $g$ of this case. Applying Lemma $A$ with

$$
F(z)=\frac{z f^{\prime}(z)}{K(z)-K(-z)}
$$

we easily get after some simplification as in Case 1 that

$$
\frac{z(h * f)^{\prime}(z)}{(h * K)(z)-(h * K)(-z)}
$$

is contained in the convex hull of $F$. That is

$$
\operatorname{Re} \frac{z(h * f)^{\prime}(z)}{(h * K)(z)-(h * K)(-z)}>0, \quad z \in U
$$

Thus $\mathrm{h} * \mathrm{f} \in \mathrm{K}_{\mathrm{S}}$ provided $\mathrm{h} * \mathrm{~K} \in \mathrm{S}_{\mathrm{S}}^{*}$. However, the latter is true as seen by Case 1. CASE 4. Let $f \in S_{s}^{*}(\lambda), 0<\lambda \leq 1$, and $h \in C$. It is clear from (1.3) and (1.2) that $S_{S}^{*}(\lambda), S_{s}^{*}$ for all $0<\lambda<1$. Let $g$ and $F$ as defined in Case 1 , then, as in the proof of Case 1,

$$
\frac{z(h * f)^{\prime}(z)}{(h * f)(z)-(h * f)(-z)}
$$

lies in the convex hu11 of $F$. This means

$$
\left|\arg \frac{z(h * f)^{\prime}(z)}{(h * f)(z)-(h * f)(-z)}\right|<\lambda \pi / 2,0<\lambda<1
$$

Thus $\mathrm{h} * \mathrm{f}, \mathrm{S}_{\mathrm{s}}{ }^{(}(\lambda)$ by (1.3). This completes the proof of Theorem 1 .

3. APPLICATIONS

For $X$, let $r_{C}(X)$ denote the largest positive number so that every $f, X$ 
is convex in $|z|<r_{c}(X)$. Consequently, the conclusion of Theorem 1 remains valid for $h * f$ in the disk $|z|<r_{c}(X)$ for each $h \in X$ and every $f$ in the given four classes. This observation was first used effectively by Barnard and Kellogg in [1] to unify and reduce the amount of work usually associated with such problems. Let

$$
\begin{aligned}
h_{\gamma}(z) & =\sum_{n=1}^{\infty} \frac{\gamma+n}{\gamma+1} z^{n}=\frac{\gamma}{1+\gamma} \frac{z}{1-z}+\frac{1}{1+\gamma} \frac{z}{(1-z)^{2}}, \\
H(z) & =\sum_{n=1}^{\infty} \frac{\gamma+1}{\gamma+n} z^{n}, \text { and } \\
P(z) & =\sum_{n=1}^{\infty} \frac{1-x^{n}}{(1-x) n} z^{n}=\frac{1}{1-x} \log \frac{1-x z}{1-z},
\end{aligned}
$$

where $\operatorname{Rer} \geq 0,|x|=1, x \neq 1$ and for all $z$ in $U$. The operators $B, j=1,2,3$, defined below have been studied by many authors. Let

$$
\begin{aligned}
& B_{1} f(z)=\frac{\gamma f(z)+z f^{\prime}(z)}{1+\gamma} \equiv\left(h_{\gamma} * f\right)(z), \operatorname{Re} \gamma=0, \\
& B_{2} f(z)=\frac{1+\gamma}{z^{\gamma}} \int_{0}^{z} t^{\gamma-1} f(t) d t=\left(H_{\gamma} * f\right)(z), \text { Re } \gamma \geq 0, \\
& B_{3} f(z)=\int_{0}^{z} \frac{f(t)-f(x t)}{t-x t} d t=(P * f)(z),|x| \leq 1, x \neq 1 .
\end{aligned}
$$

For example, the operator $B_{1}$ is due to Livingston [7] when $\gamma=1$, $B_{2}$ is due to Libera [5] when $\gamma=1$ while $B_{3}$ was first used by Pommerenke in [10], see also [2], i3], [6], [11]. Consequently, the operators $B_{j}, j=1,2,3$, preserve the classes $S_{s}{ }^{*}, C_{s}, K_{s}$ or $S_{s} *(\lambda)$ up to $r_{c}\left(h_{\gamma}\right), r_{c}\left(H_{\gamma}\right)$ or $r_{c}(P)$ as mentioned at the start of this section. However, $r_{c}\left(H_{\gamma}\right)=1$, Rer $\geq 0$ was shown by Ruscheweyh [11], and $r_{c}(P)=1$ was shown by Pommerenke [10]. We need only to calculate $r_{c}\left(h_{\gamma}\right)$. From the definition of $h_{\gamma}$ we get

$$
h_{\gamma}{ }^{\prime}(z)=\frac{1+\frac{1-\gamma}{1+\gamma} z}{(1-z)^{3}}=\frac{1+c z}{(1-z)^{3}}
$$

where $c=\frac{1-\gamma}{1+\gamma}$. Thus

$$
1+\frac{z h_{\gamma}^{\prime \prime}(z)}{h_{\gamma}^{\prime}(z)}=\frac{2+z}{1-z}-\frac{1}{1+c z} .
$$

This implies

$$
\operatorname{Re}\left(1+\frac{z h_{\gamma}^{\prime \prime}(z)}{h_{\gamma}^{\prime}(z)}\right) \geq \frac{2-r}{1+r}-\frac{1}{1-|c| r},|z|=r
$$

This shows that $r_{c}\left(h_{\gamma}\right)$ is the smallest positive number $r$ satisfying

$$
|c| r^{2}-2(1+|c|) r+1=0
$$

or

$$
r_{c}\left(h_{\gamma}\right)=\frac{1}{1+|c|+\sqrt{1+|c|+|c|}} .
$$


Finally, we have the following theorem.

THEOREM 2. If $\mathrm{f}$ is in $\mathrm{s}_{\mathrm{s}}{ }^{*}, \mathrm{C}_{\mathrm{s}}, \mathrm{K}_{\mathrm{s}}$ or $\mathrm{s}_{\mathrm{s}}{ }^{*}(\lambda), 0<\lambda \leqslant 1$, then

i) $B_{j} f$ is in $S_{s}^{*}, C_{s}, K_{S}$ or $S_{s}{ }^{*}(\lambda)$, respectively for each $j=2,3$, and

ii) $\mathrm{B}_{1} \mathrm{f}$ is in $\mathrm{S}_{\mathrm{S}}{ }^{*}, \mathrm{C}_{\mathrm{S}}, \mathrm{K}_{\mathrm{S}}$ or $\mathrm{S}_{\mathrm{S}}{ }^{*}(\lambda)$ respectively for

$$
\begin{aligned}
& |z|<\mathrm{r}_{\mathrm{c}}\left(\mathrm{h}_{\gamma}\right)=1 /\left(1+|\mathrm{c}|+\sqrt{1+|\mathrm{c}|+|\mathrm{c}|^{2}}\right), \quad c=\frac{1-\gamma}{1+\gamma}, \\
& \text { R. } \gamma \geq 0 .
\end{aligned}
$$

REMARK 4. Theorem 2 part (i) was shown in [8] for the class $s_{s}^{*}(\lambda)$ on $1 y$. Part (i) with $\gamma=1$ and $x=-1$ was shown in [4] for the classes $S_{S}^{*}, C_{s}$ and $K_{S}$.

\section{REFERENCES}

1. BARNARD, R. W., KELLOGG, C., Applications of convolution operators to problems in univalent function theory, Michigan Math. J., 27 (1980), 81-94.

2. BARINARDI, S. D., Convex and Starlike univalent functions, Trans. Amer. Math. Soc. 135 (1969), 429-446.

3. CAUSEY, W. M., The close to convexity and univalence of an integral, Math. Z. 99 (1967), 207-212.

4. DAS, R. N. and SINGH, P., On subclasses of schlicht mappings, Indian J. Pure Appl. Math. 8 (1977), 864-872.

5. LIBERA, R. J., Some classes of regular univalent functions, Proc. Amer. Math. Suc. 16 (1965), 775-758.

6. LIBERA, R. J. and LIVINGSTON, A. E., On the univalence of some classes of regular functions, Proc. Amer. Math. Soc. 30 (1971), 327-336.

7. LIVINGSTON, A. E., On the radius of univalence of certain analytic functions, Proc. Amer. Math. Soc. 17 (1966), 352-357.

8. PADMANABHAN, K. S. and THANGAMANI, J., The effect of certain integral operators on some classes of starlike functions with respect to symmetric points, Bull. Math. de 1a Soc. Sci. Math. de 1a R.S, de Rawmanie 25 (1982), 355-360.

9. PólyA, G. and SCHOENBERG, I. J, Remarks on de la Vallée Poussin means and convex conformal maps of the circle, Pacific J. Math. $\underline{8}$ (1958), 295-334.

10. POMMERENKE, C., On close-to-convex analytic functions, Trans. Amer. Math. Soc. 114 (1965), 176-186。

11. RUSCHEWEYH, S., New criteria for univalent functions, Proc. Amer. Math. Soc. 49 (1975), 109-115.

12. RUSCHEWEYH, $S$ and SHEIL-SMALL, T., Hadamard products of schlicht functions and the Pólya-Schoenberg conjecture, Comment. Math. Helv. 48 (1973), 119-135.

13. SAKAGUCHI, K., On a certain univalent mapping, J. Math. Soc. Japan 11 (1959),72-80. 


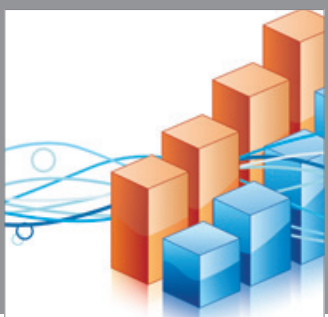

Advances in

Operations Research

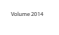

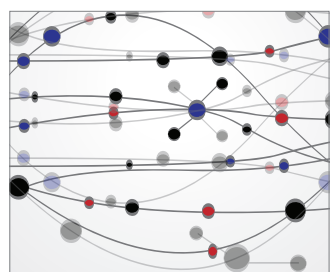

\section{The Scientific} World Journal
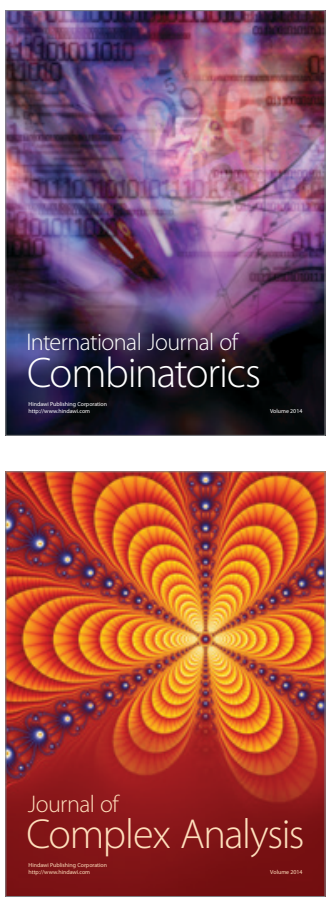

International Journal of

Mathematics and

Mathematical

Sciences
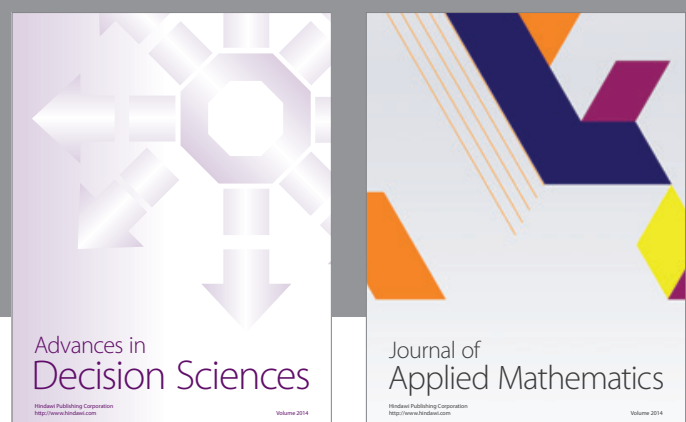

Journal of

Applied Mathematics
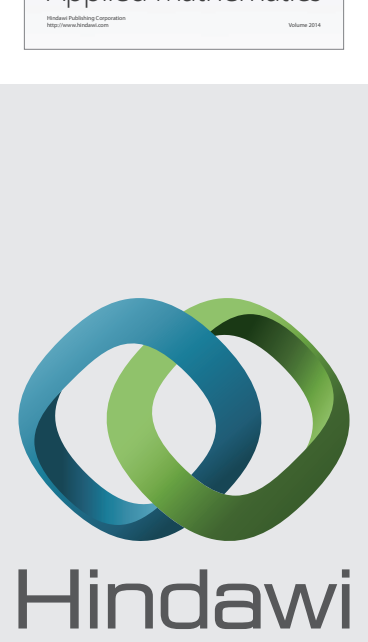

Submit your manuscripts at http://www.hindawi.com
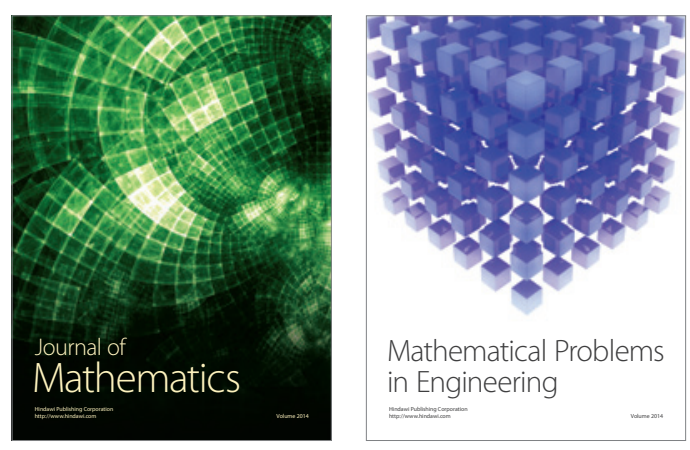

Mathematical Problems in Engineering
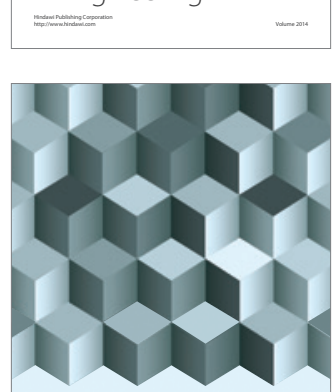

Journal of

Function Spaces
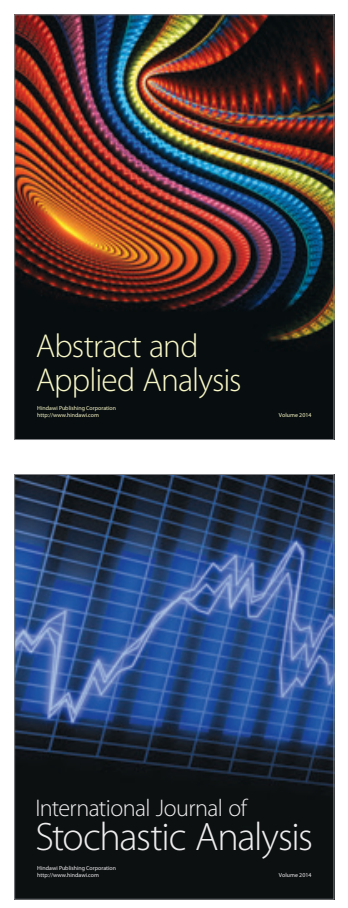

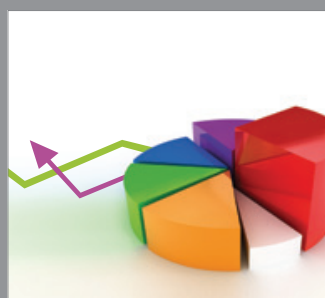

ournal of

Probability and Statistics

Promensencen
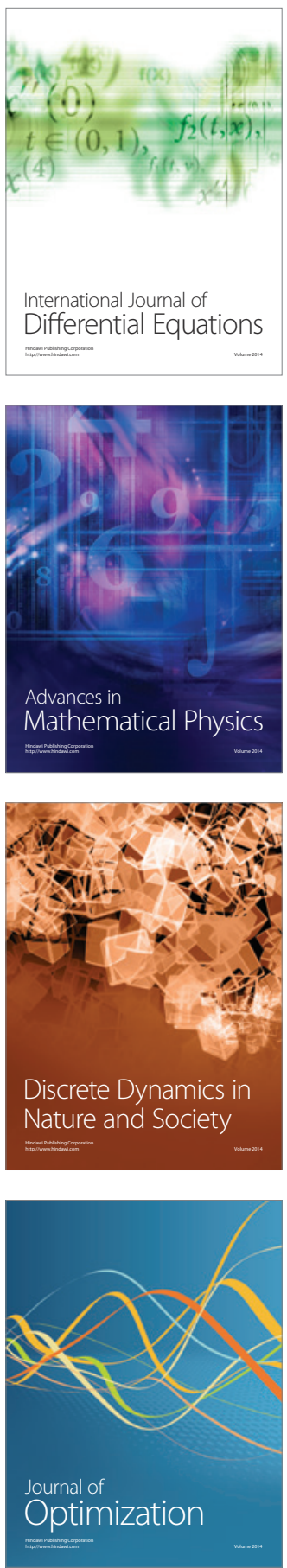